\title{
Auch ältere Patienten profitieren von der Chemotherapie
}

Davidoff AJ et al. Chemotherapy and survival benefit in elderly patients with

advanced non-small-cell lung cancer. J Clin Oncol. 2010;28:2191-2197

\section{Fragestellung}

Eine Arbeitsgruppe des Greenebaum Cancer Center, Baltimore (USA) untersuchte retrospektiv den Effekt chemotherapeutischer Therapieregimes auf das Überleben älterer Patienten mit fortgeschrittenem nicht kleinzelligem Bronchialkarzinom (NSCLC).

\section{Methodik}

Die Daten von 21285 Patienten (Alter $\geq 65$ Jahren) mit fortgeschrittenem NSCLC (13,9\% im Stadium IIIB und $76,9 \%$ im Stadium IV) wurden in die Studie aufgenommen. Sie stammten aus einer großen Datenbank von 16 onkologischen Zentren (National Cancer Institute Surveillance, Epidemiology and End Results Database, SEER).

\section{Ergebnisse}

Nur 30,1\% der Patienten erhielten eine antineoplastische Chemotherapie. Eine Zweitlinientherapie erhielten 37,1\%. Zwei Drittel der Patienten erhielten eine platinhaltige Kombinationstherapie (da- von $72 \%$ eine Kombination mit einem Taxan) und 23,3\% eine Monotherapie (bevorzugt Patienten über 80 Jahren). Carboplatin wurde deutlich häufiger eingesetzt als Cisplatin. Patienten mit einer platinhaltigen Kombinationstherapie erhielten häufiger eine Zweitlinientherapie (59,1\% als Monotherapie).

Es zeigte sich ein klarer Überlebensvorteil für Patienten, die eine Chemotherapie erhielten $(7,1$ vs. 2,5 Monate; $\mathrm{p}<$ 0,001). Das 1-Jahres-Überleben betrug $11,6 \%$ ohne Chemotherapie und 27\% mit Chemotherapie (Abb. 1).

\section{Kommentar}

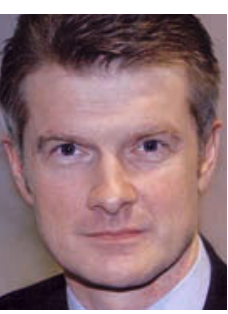

Prof. Dr. med. Martin Kohlhäufl, Stuttgart-Gerlingen

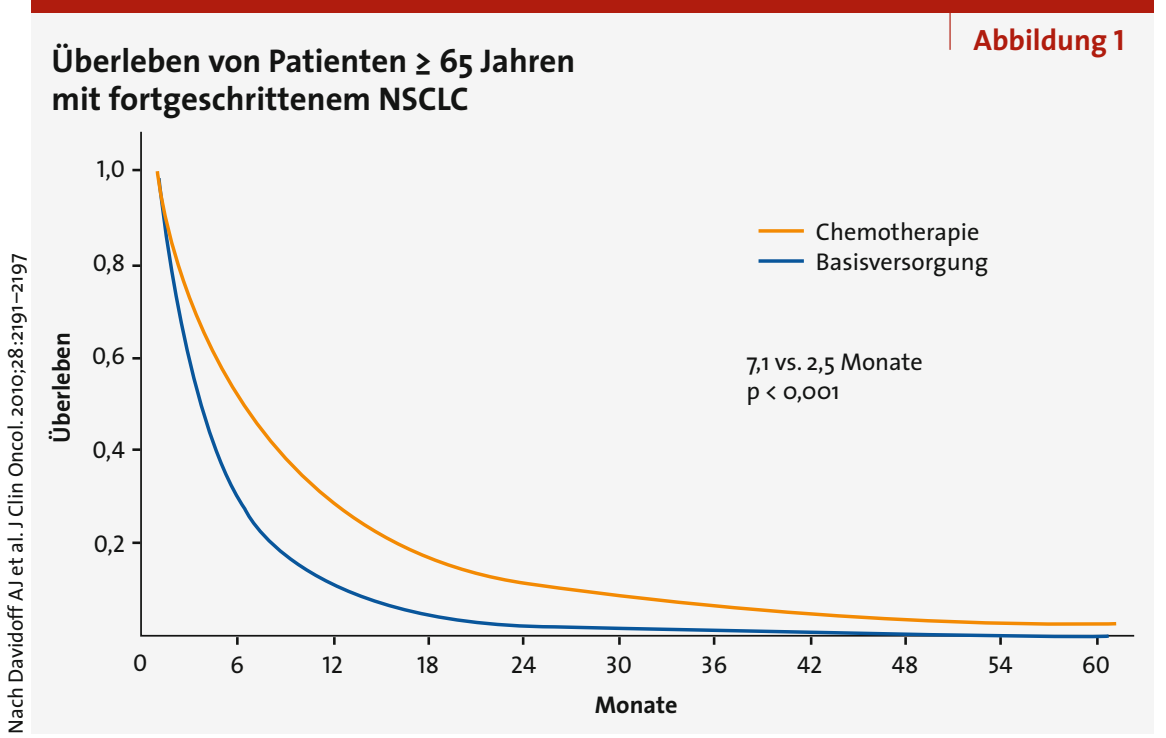

Diese retrospektive Analyse zeigt klar, dass die meisten Patienten mit NSCLC im höheren Alter keine antineoplastische Chemotherapie erhalten, obwohl sie das Überleben signifikant verlängert. Die 1-JahresÜberlebensrate von älteren Patienten, die eine platinhaltige Kombinationstherapie erhielten, war nach der Meinung der Autoren vergleichbar mit den Überlebensraten in aktuellen Studien zur Therapie des fortgeschrittenen NSCLC.

Aus retrospektiven Analysen ist bekannt, dass das Alter per se kein unabhängiger Prognosefaktor für ein ungünstiges Ergebnis bei der Behandlung des Bronchialkarzinoms ist. Dagegen ist der Komorbiditätsindex (Charlson-Comorbidity-Score) für die Prognose bedeutsamer [3].

Zum Effekt einer Zweitlinientherapie bei älteren Patienten konnte die Studie aus methodischen Gründen nicht Stellung nehmen, ebenso nicht zu Fragen der Toxizität und Lebensqualität älterer Patienten unter einer Chemotherapie. Der soziale Rückhalt erschien von großer Bedeutung, da verheiratete Patienten signifikant häufiger eine antineoplastische Therapie erhielten.

\section{Fazit}

Für wichtige Therapieentscheidungen bei älteren Patienten mit einem Bronchialkarzinom fehlt immer noch eine Datenbasis mit hohem Evidenzniveau, da ältere Patienten bislang in klinischen Studien deutlich unterrepräsentiert waren.

Im praktischen Alltag ist ein hohes Alter unabhängig von den Komorbiditäten immer noch ein wichtiges Entscheidungskriterium gegen den Einsatz einer antineoplastischen Chemotherapie - trotz gegenteiliger Studienerkenntnisse [4]. In Zukunft werden wir durch die konsequente Einbeziehung eines geriatrischen Assessments in klinische Studien verlässliche Kriterien zurVerfügung haben, die Aussagen darüber erlauben, welcher ältere Patient von welcher Therapie am ehesten profitieren wird.

\footnotetext{
Literatur

1. Edwards BK et al. Cancer 2002;94:2766-2792 2. Hurria A et al. Crit Rev Oncol Hematol. 2007;61:255260

3. Asmis TR et al. J Clin Oncol. 2008;26:54-59 4. Gridelli C et al. Lung Cancer 2009;66:282-286
} 